\title{
A DECLINE IN THE NONTHERMAL X-RAY EMISSION FROM CASSIOPEIA A
}

\author{
Daniel J. Patnaude ${ }^{1}$, Jacco Vink ${ }^{2}$, J. Martin Laming ${ }^{3}$, and Robert A. Fesen ${ }^{4}$ \\ ${ }^{1}$ Smithsonian Astrophysical Observatory, Cambridge, MA 02138, USA \\ 2 Astronomical Institute, Utrecht University, P.O. Box 80000, 3508 TA Utrecht, The Netherlands \\ ${ }^{3}$ Space Science Division, Naval Research Laboratory, Code 7674L, Washington, DC 20375, USA \\ ${ }^{4} 6127$ Wilder Lab, Department of Physics \& Astronomy, Dartmouth College, Hanover, NH 03755, USA \\ Received 2010 November 29; accepted 2011 February 7; published 2011 February 18
}

\begin{abstract}
We present new Chandra ACIS-S3 observations of Cassiopeia A which, when combined with earlier ACIS-S3 observations, show evidence for a steady $\sim 1.5 \%-2 \% \mathrm{yr}^{-1}$ decline in the $4.2-6.0 \mathrm{keV}$ X-ray emission between the years 2000 and 2010. The computed flux from exposure corrected images over the entire remnant showed a $17 \%$ decline over the entire remnant and a slightly larger $(21 \%)$ decline from regions along the remnant's western limb. Spectral fits of the 4.2-6.0 keV emission across the entire remnant, forward shock filaments, and interior filaments indicate that the remnant's nonthermal spectral power-law index has steepened by about $10 \%$, with interior filaments having steeper power-law indices. Since TeV electrons, which give rise to the observed X-ray synchrotron emission, are associated with the exponential cutoff portion of the electron distribution function, we have related our results to a change in the cutoff energy and conclude that the observed decline and steepening of the nonthermal X-ray emission is consistent with a deceleration of the remnant's $\simeq 5000 \mathrm{~km} \mathrm{~s}^{-1}$ forward shock of $\approx 30-70 \mathrm{~km} \mathrm{~s}^{-1} \mathrm{yr}^{-1}$.
\end{abstract}

Key words: acceleration of particles - ISM: individual objects (Cassiopeia A) - radiation mechanisms: non-thermal

Online-only material: color figures

\section{INTRODUCTION}

Supernova remnants (SNRs) have long been considered to be the primary source of Galactic cosmic rays (CRs) below the knee of the cosmic-ray spectrum, $\sim 10^{15} \mathrm{eV}$. TeV $\gamma$-ray observations of SNRs such as RX J1713.7-3946 and RX J0852.0-4622 provide evidence for the acceleration of ions (Aharonian et al. 2007a, 2007b). However, the TeV emission can also be attributed to inverse Compton scattering by the same electron population that produces the X-ray synchrotron emission.

Viewed in X-rays, the young ( 330 yr; Fesen et al. 2006) Galactic SNR Cassiopeia A (Cas A) consists of a shell whose emission is dominated by emission lines from $\mathrm{O}, \mathrm{Si}, \mathrm{S}$, and $\mathrm{Fe}$ (e.g., Vink et al. 1996; Hughes et al. 2000; Willingale et al. 2002, 2003; Hwang \& Laming 2003; Laming \& Hwang 2003). Exterior to this shell are faint X-ray filaments which mark the location of the forward shock. The emission found here is nonthermal X-ray synchrotron emission from shock accelerated electrons (Allen et al. 1997; Gotthelf et al. 2001; Vink \& Laming 2003). These forward shock filaments are observed to expand with a velocity of $\simeq 5000 \mathrm{~km} \mathrm{~s}^{-1}$ (DeLaney \& Rudnick 2003; Patnaude \& Fesen 2009), assuming an SNR distance of $3.4 \mathrm{kpc}$ (Reed et al. 1995).

Nonthermal emission filaments are also observed in the interior of the SNR and are believed to be either a forward shock seen in projection (DeLaney et al. 2004; Patnaude \& Fesen 2009) or associated with efficient acceleration of electrons at the reverse shock (Uchiyama \& Aharonian 2008; Helder \& Vink 2008). Fluctuations in both exterior and interior nonthermal filaments have also been reported (Patnaude \& Fesen 2007, 2009; Uchiyama \& Aharonian 2008), and the variability is cited as evidence for rapid synchrotron cooling of $\mathrm{TeV}$ electrons in $\mathrm{mG}$-scale fields. A 2-4 year timescale for variations is evidence for diffusive shock acceleration in SNR shocks, or alternatively the variations are seen as evidence for magnetic field fluctuations due to plasma waves behind the shock (Bykov et al. 2008).
Emission from Cas A has been seen at energies up to $\sim 40 \mathrm{keV}$ with the Suzaku HXD PIN detector (Maeda et al. 2009), up to $100 \mathrm{keV}$ with the Compton Gamma Ray Observatory OSSE and Integral IBIS (The et al. 1996; Renaud et al. 2006), and GeV emission has been detected using Fermi-LAT (Abdo et al. 2010). The Fermi observations do not rule out either a leptonic or hadronic origin to the $\mathrm{GeV}$ emission. Finally, Cas A has been detected at TeV energies with HEGRA, MAGIC, and Veritas (Aharonian et al. 2001; Albert et al. 2007; Humensky 2008). Interestingly, the centroids for the GeV-TeV emission are located in the western region of Cas A, where the nonthermal X-ray emission is brightest (Helder \& Vink 2008; Maeda et al. 2009).

Here we present Chandra ACIS-S3 observations of Cas A taken in 2009 and 2010 which, when compared to ACIS-S3 observations taken between 2000 and 2007, show the remnant's nonthermal X-ray emission in the $4.2-6.0 \mathrm{keV}$ band to have decreased at a rate of $\simeq 1.5 \%-2.0 \% \mathrm{yr}^{-1}$. In Section 2 we discuss our observations, data reduction, and spectral analysis and in Section 3 we discuss our results and offer some conclusions about the current and future evolution of the nonthermal emission in Cas A.

\section{OBSERVATIONS, DATA REDUCTION, AND ANALYSIS}

Cas A has been observed extensively with Chandra and we have made use of several GO observations taken between 2000 and 2010, including the 2004 VLP (PI: Hwang). We reprocessed each epoch data set listed in Table 1 using CIAO $^{5}$ version 4.2 and CalDB 4.2.2. The more recent observations were split due to spacecraft thermal constraints. We merged these split observations into a single event list for each epoch.

We filtered the events on energies between 4.2 and $6.0 \mathrm{keV}$ and performed an exposure correction assuming a monochromatic $5.1 \mathrm{keV}$ source. The exposure corrected image is in

\footnotetext{
5 http://chandra.harvard.edu
} 


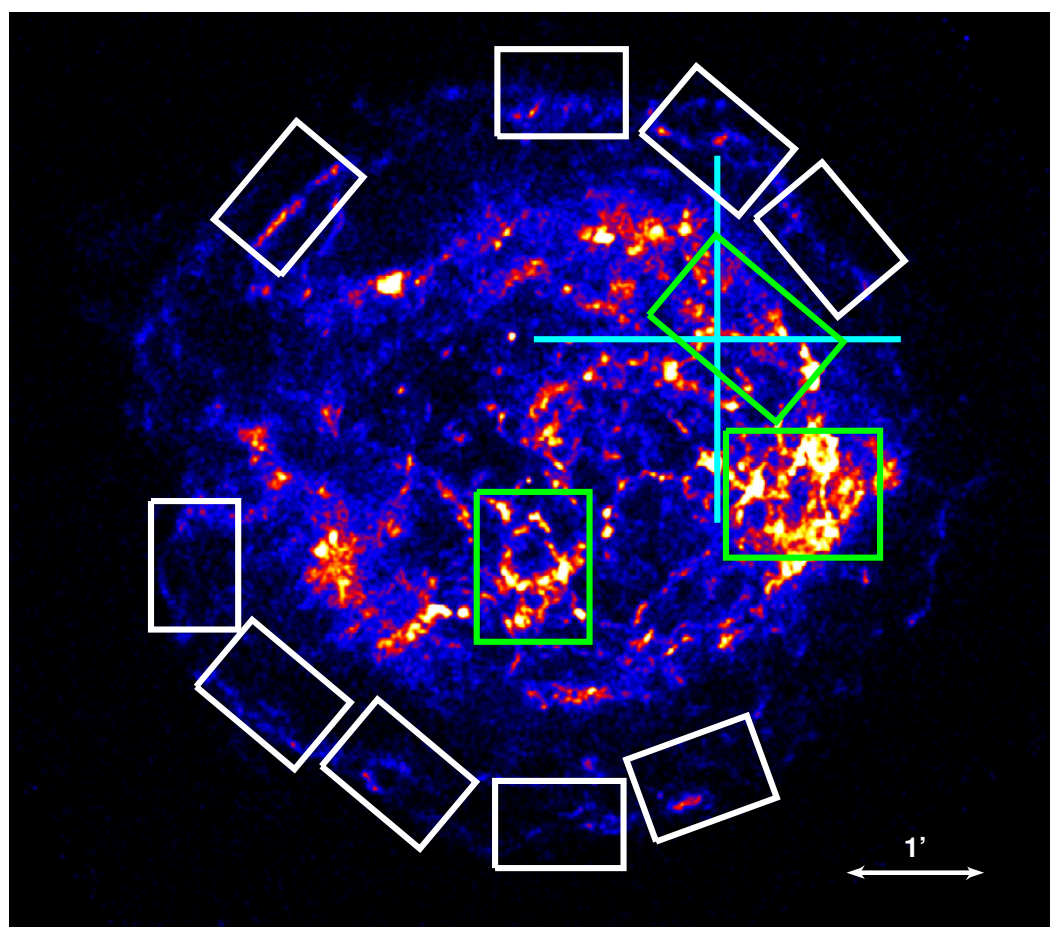

Figure 1. Exposure corrected image of Cas A between 4.2 and $6.0 \mathrm{keV}$. The white boxes mark approximately the locations of the spectra extracted from the forward shock, while the green boxes mark those regions where spectra were extracted from the interior filaments. The cyan cross marks the location and $68 \%$ confidence limit of the Fermi centroid.

(A color version of this figure is available in the online journal.)

Table 1

Chandra Observations of Cas A and Spectral Fitting Results

\begin{tabular}{|c|c|c|c|c|c|c|}
\hline $\begin{array}{l}\text { Epoch } \\
(\mathrm{yr})\end{array}$ & $\overline{\Gamma_{\mathrm{SNR}}}$ & $\begin{array}{c}F^{\mathrm{a}} \\
\left(10^{-10} \mathrm{erg} \mathrm{cm}^{-2} \mathrm{~s}^{-1}\right)\end{array}$ & $\Gamma_{\mathrm{FS}}$ & $\overline{\Gamma_{\text {Interior }}}$ & $\overline{\Gamma_{\text {West }}}$ & $\begin{array}{c}F_{\text {West }^{\mathrm{a}}} \\
\left(10^{-10} \mathrm{erg} \mathrm{cm}^{-2} \mathrm{~s}^{-1}\right)\end{array}$ \\
\hline 2000.1 & $2.81 \pm 0.03$ & $1.61 \pm 0.01$ & $2.32 \pm 0.11$ & $2.66 \pm 0.07$ & $2.66 \pm 0.06$ & $0.229 \pm 0.001$ \\
\hline 2002.1 & $3.01 \pm 0.03$ & $1.56 \pm 0.01$ & $2.43 \pm 0.11$ & $2.75 \pm 0.07$ & $2.74 \pm 0.06$ & $0.223 \pm 0.001$ \\
\hline 2004.1 & $2.99 \pm 0.03$ & $1.54 \pm 0.01$ & $2.42 \pm 0.11$ & $2.70 \pm 0.07$ & $2.73 \pm 0.06$ & $0.215 \pm 0.001$ \\
\hline 2007.9 & $2.98 \pm 0.04$ & $1.45 \pm 0.02$ & $2.55 \pm 0.15$ & $2.70 \pm 0.10$ & $2.80 \pm 0.09$ & $0.197 \pm 0.002$ \\
\hline 2009.8 & $2.99 \pm 0.05$ & $1.42 \pm 0.04$ & $2.61 \pm 0.15$ & $2.78 \pm 0.11$ & $2.78 \pm 0.09$ & $0.195 \pm 0.004$ \\
\hline 2010.8 & $3.07 \pm 0.04$ & $1.34 \pm 0.01$ & $2.56 \pm 0.14$ & $2.82 \pm 0.13$ & $2.85 \pm 0.08$ & $0.183 \pm 0.002$ \\
\hline
\end{tabular}

Note. ${ }^{\text {a }} 4.2-6.0 \mathrm{keV}$ flux.

units of photons $\mathrm{cm}^{-2} \mathrm{~s}^{-1}$ pixel ${ }^{-1}$, so to compute the flux of $5.1 \mathrm{keV}$ photons $\mathrm{cm}^{-2} \mathrm{~s}^{-1}$, we integrate over the number of pixels. The latest data set, taken in 2010 November, is shown in Figure 1.

As seen in the black curve in Figure 2, there is a decrease in the overall 4.2-6.0 keV emission from Cas A. This decline does not appear to be instrumental in nature. Several sources have been observed as Chandra calibration targets, including the cluster A1795. In Figure 2, we plot the 4.2-6.0 keV emission from A1795, showing that the emission from this cluster has not varied by more than $1 \%-2 \%$ over $\sim 10$ years (A. Vikhlinin 2010, private communication). As a further check on whether our result for Cas A could be due to an instrumental artifact, we plot in Figure 2 the 1.5-3.0 keV emission (exposure corrected at $1.85 \mathrm{keV}$ ) and find that emission in that band has declined by less than $1 \%$ in 11 years. In addition, the Galactic SNR, G21.5-0.9, has been observed extensively with Chandra ACIS-S3 as a steady $2.0-8.0 \mathrm{keV}$ continuum source, and changes in the flux from that source are not observed at the level which we report here (Tsujimoto et al. 2011). Finally, we note that Katsuda et al. (2010) reported no change in the X-ray synchrotron emission from SN 1006.
As an additional test of this flux decline, we fitted the 4.2 $6.0 \mathrm{keV}$ continuum emission in $\mathrm{Cas} \mathrm{A}$ at each epoch to a powerlaw model in $\mathrm{XSPEC}^{6}$ version 12.6. We find that the modeled flux does decrease with time in this energy band, consistent with our analysis of the exposure corrected images. As shown in Column 2 of Table 1, the fitted spectrum appears to steepen with time.

The results of our spectral fits listed in Column 2 of Table 1 are across the entire SNR and thus can include emission from electrons accelerated at the remnant's forward shock, the reverse shock, or at the contact interface. To determine whether the changing emission is from the outer forward shock filaments, interior regions or possibly both, we extracted spectra from several regions marked with boxes in Figure 1 in all data sets and again fit the nonthermal emission to a power-law model. Galactic absorption has a negligible effect on $5.0 \mathrm{keV}$ photons, so we did not model it here.

In Columns 4-6 of Table 1, we list the results from the spectral fits for the forward shock regions (the white boxes in Figure 1), the interior regions (green boxes in Figure 1), and

\footnotetext{
6 http://heasarc.gsfc.nasa.gov/docs/xanadu/xspec/
} 


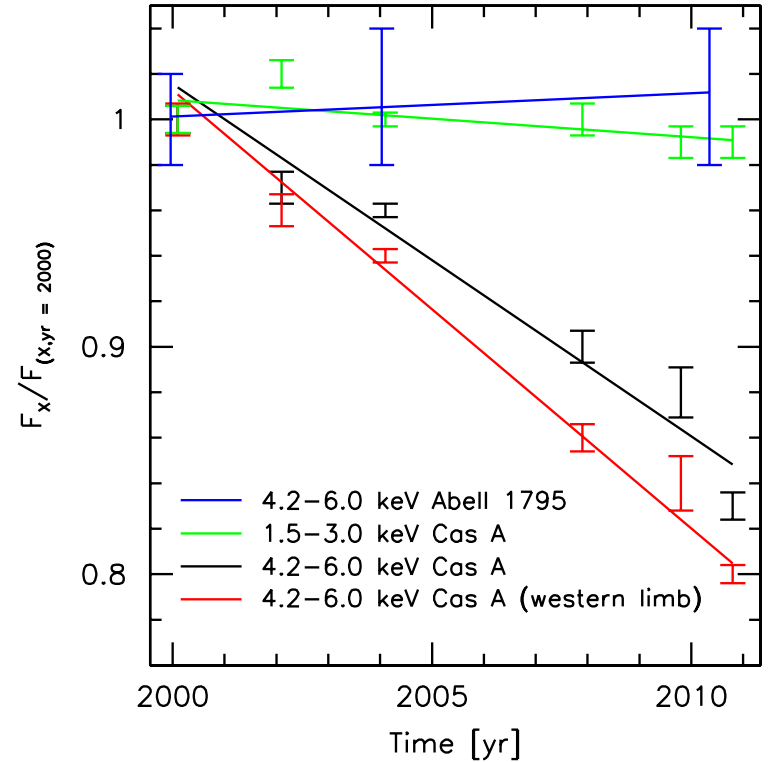

Figure 2. Comparison of 4.2-6.0 keV flux in Cas A compared to the year 2000 observations. The black curve and data correspond to changes in the whole SNR, while the red curve and data correspond to changes in the western portion of Cas A only. For reference we also show the 1.5-3.0 keV flux from Cas A (fluxed at $1.85 \mathrm{keV}$ ) as well as the $4.2-6.0 \mathrm{keV}$ emission from the cluster A1795. The observed decline in the 4.2-6.0 keV emission in Cas A corresponds to a fractional decline of $-(1.5 \pm 0.17) \% \mathrm{yr}^{-1}$ across the whole SNR and $-(1.9 \pm$ $0.10) \% \mathrm{yr}^{-1}$ in the western limb.

(A color version of this figure is available in the online journal.)

then also just the interior regions that coincide with the Fermi centroid (Abdo et al. 2010). As can be seen in Table 1, the spectral shape of the forward shock filaments varies with time, with $\Gamma_{\mathrm{FS}} \sim 2.3-2.6$ between 2000.1 and 2010.8. The interior filaments and the region associated only with the Fermi centroid in Figure 1 also show an increase in the power-law index over the same time period. Interior filaments show a steeper spectral shape than that seen for forward shock regions. We also examined changes in intensity in the western portion of Cas A and found that the emission from this region is also decreasing with time. This is shown as the red curve in Figure 2 and is seen to be steeper than the remnant's overall nonthermal emission decline rate (black curve).

\section{DISCUSSION}

As shown in Figure 2 and listed in Table 1, we find a decline in the 4.2-6.0 keV emission in Cas A over an approximately 11 year span. A linear least-squares fit to the fractional change in 4.2-6.0 keV emission indicates a fairly steady decline in the nonthermal emission of $(1.5 \pm 0.17) \% \mathrm{yr}^{-1}$ across the entire SNR. In western regions coincident with the Fermi centroid, the rate of decline is slightly higher at $(1.9 \pm 0.1) \% \mathrm{yr}^{-1}$.

A much slower decline in Cas A's radio emission from $\mathrm{GeV}$ electrons has been known for some time (Shklovskii 1960; Baars et al. 1977). Reichart \& Stephens (2000) report that Cas A has generally been fading at a rate of $\sim 0.6 \%-0.7 \% \mathrm{yr}^{-1}$ over a range of radio frequencies. The observed decline in Cas A's radio emission has been interpreted to be a result of adiabatic expansion of the SNR since radio synchrotron emitting electrons have no appreciable radiative losses (Shklovskii 1960; Anderson \& Rudnick 1996).

In contrast, the remnant's X-ray synchrotron emission is the result of electrons accelerated within the last decade, as their radiative loss times are of the order 10 years, or possibly less.
Thus, X-ray synchrotron emission is much more sensitive to the present-day acceleration time and radiative losses. For typical SNR parameters, synchrotron X-rays are produced in large part by the exponential tail of the electron distribution (e.g., Ellison \& Cassam-Chenaï 2005). Thus, any energy loss will result in a large drop in the emissivity, such as observed here in Cas A.

In order to interpret the $4.2-6.0 \mathrm{keV}$ flux changes seen in Cas A's X-ray continuum emission, we now examine how much of this change can be attributed to a change in the cutoff energy alone, as this influences both the slope of the spectrum and the flux for photon energies above the cutoff energy.

The X-ray synchrotron spectrum near the cutoff is generally approximated by a power-law continuum with an exponential cutoff (Reynolds 1998). Zirakashvili \& Aharonian (2007) noted that in the case of a loss-limited spectral cutoff, as is likely the case for Cas A (Vink \& Laming 2003), the exponential cutoff falls off roughly as $\exp \left(-\sqrt{E / E_{c}}\right)$. We therefore use as an approximation for the photon spectrum (see also Katsuda et al. 2010)

$$
N(E)=\phi B^{\Gamma_{0}} \times E^{-\Gamma_{0}} \exp \left(-\sqrt{\frac{E}{E_{c}}}\right),
$$

where $E$ is the energy of the emitted photon, which here we assume to be $5.1 \mathrm{keV}, E_{c}$ is the cutoff energy in $\mathrm{keV}$, and $\Gamma_{0}$ is the slope of the power law well below the cutoff energy. We have split here the normalization constant into two factors, for reasons that we will explain below. One factor directly corresponds to the downstream magnetic field strength (cf. Ginzburg \& Syrovatskii 1965 , Equation (3.31)), where $\phi$ relates to the overall electron acceleration efficiency. Equation (1) implies that the spectral slope $\Gamma$ in X-rays is given by (Vink et al. 1999)

$$
\Gamma=\Gamma_{0}+\frac{1}{2} \sqrt{\frac{E}{E_{c}}} .
$$

Thus, the measured spectral index $\Gamma$ can be related to the cutoff energy by

$$
E_{c}=\frac{E}{4\left(\Gamma-\Gamma_{0}\right)^{2}} .
$$

For $\Gamma_{0}$, we assume the value inferred from the radio spectral index, namely $\Gamma_{0}=\alpha+1=1.78$ (Baars et al. 1977), or if nonlinear effects are important, $\Gamma_{0}$ may be as low as 1.25 , corresponding to a particle spectral index of 1.5 (Malkov \& O'C Drury 2001). For $\Gamma_{0}=1.5, E_{c}=1.0-2.0 \mathrm{keV}$, assuming $\Gamma=2.6-2.3$ and $E=5.1 \mathrm{keV}$. Note that Zirakashvili \& Aharonian (2007) adopt a rather steep spectrum (their Equation (35), corresponding to $\Gamma_{0}=2$ ), but this applies to the synchrotron spectrum from the remnant as a whole. In that the spectrum comes steeper, as far downstream one expects a synchrotron cooling break. This should not apply to the narrow filaments associated with shock fronts.

Differentiating Equation (2) with respect to time under the assumption that only the cutoff energy varies leads to

$$
\frac{d E_{c}}{d t}=-4 \sqrt{\frac{E_{c}}{E}} E_{c} \frac{d \Gamma}{d t} .
$$

According to Table 1 , we measure $d \Gamma / d t=0.018 \mathrm{yr}^{-1}$ in the western portion of Cas $\mathrm{A}$, which translates to $d E_{c} / d t=$

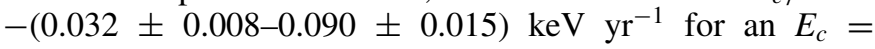
1.0-2.0 keV. Likewise, from Table 1 for the forward shock, 
$d \Gamma / d t=0.022 \mathrm{yr}^{-1}$, which implies $d E_{c} / d t=-(0.039 \pm$ $0.004-0.101 \pm 0.008) \mathrm{keV} \mathrm{yr}^{-1}$.

That the cutoff energy is changing is likely due to the deceleration of the shock. For example, if we use Equation (34) of Zirakashvili \& Aharonian (2007) with $\kappa=$ $1 / \sqrt{11}$ and $\gamma_{s}=4$, we obtain for the cutoff energy

$$
E_{c} \approx 1.3 \eta^{-1}\left(\frac{V_{s}}{3000 \mathrm{~km} \mathrm{~s}^{-1}}\right)^{2} \mathrm{keV},
$$

where $\eta$ is the diffusion parameter, and equals 1 in the case of Bohm diffusion. Therefore, the change in cutoff energy relates to a change in shock velocity as

$$
\frac{d V_{s}}{d t}=3.5 \times 10^{6}\left(\frac{V_{s}}{\mathrm{~km} \mathrm{~s}^{-1}}\right)^{-1} \eta \frac{d E_{c}}{d t} .
$$

The forward shock of Cas A has been measured to be $\simeq 5000 \mathrm{~km} \mathrm{~s}^{-1}$ (DeLaney \& Rudnick 2003; Patnaude \& Fesen 2009), so $d V_{s} / d t=-(27 \pm 4-71 \pm 9) \eta \mathrm{km} \mathrm{s}^{-1} \mathrm{yr}^{-1}$. This is high, as one expects for an expansion law of $R \propto t^{m}$, appropriate for Cas A $(m=0.66)$, that $d V_{s} / d t \approx 5 \mathrm{~km} \mathrm{~s}^{-1} \mathrm{yr}^{-1}$. This discrepancy could be due to inaccuracies in our assumptions. For example, a spectral cutoff that is less sharp than $\exp \left(-\sqrt{E / E_{c}}\right)$ would lead to a smaller estimate of $d V_{s} / d t$. Alternatively, it could be argued that diffusion is slower than Bohm $\eta<1$, as found in numerical simulations by Reville et al. (2008).

The nature of the reverse shock is more uncertain. Helder \& Vink (2008) have argued that the western region of Cas A has a velocity as high as $6000 \mathrm{~km} \mathrm{~s}^{-1}$, implying that the reverse shock in our frame is almost stationary. This could be the case given a possible interaction with a molecular cloud in the west (Keohane et al. 1996). However, given the uncertainties in the nature of the interior filaments and the shock velocity of the reverse shock we defer a discussion on the changes in the western region of Cas A until more observations are completed.

So far we have only interpreted the change in spectral slope as due to a change in shock velocity. In addition, using the same framework, we can interpret the observed flux changes. Differentiating Equation (1) with respect to time we obtain (cf. Katsuda et al. 2010)

$$
\begin{aligned}
\frac{d N(E)}{d t}= & E^{-\Gamma_{0}} \exp \left(-\sqrt{\frac{E}{E_{c}}}\right) \\
& \times\left[\frac{d \phi}{d t} B^{\Gamma_{0}}+\phi \frac{d B^{\Gamma_{0}}}{d t}-2 \phi B^{\Gamma_{0}} \frac{d \Gamma}{d t}\right],
\end{aligned}
$$

where we made use of Equation (4) to rewrite the last term. In what follows we will estimate the influence of each term separately.

To start with the last term describing the flux change due to the evolution of cutoff energy, it is clear from Equation (7) that a change in $E_{c}$ alone will lead to a fractional change in flux of

$$
\frac{1}{N(E)} \frac{d N(E)}{d t}=-2 \frac{d \Gamma}{d t} .
$$

This connects the change in spectral slope to the related change in flux. For the values listed above, for both the forward shock and the western region, we expect a fractional change of $\approx 4 \%$ $\mathrm{yr}^{-1}$, which is about twice the value observed.

For the other two terms in Equation (7), we make some additional assumptions about their dependence on time. Although this adds uncertainty, it is nevertheless worthwhile for making order-of-magnitude estimates. We limit our discussion to the forward shock region as both its shock evolution and density structure are better understood.

For the normalization factor $\phi$, we assume that it corresponds directly to the amount of gas entering the forward shock per unit time:

$$
\phi \propto 4 \pi r^{2} \rho V_{s} \propto t^{m-1},
$$

where we have used both the fact that the forward shock is likely to be moving through the progenitor wind of the supernova, hence $\rho \propto r^{-2}$, and also the idea that the forward shock evolution is self-similar with $r \propto t^{m}$. Note also that this equation is only valid if synchrotron losses are large, otherwise $\phi$ would be proportional to the total amount of material swept up by the shock, rather than by what is being swept up now. Differentiating the above equation with respect to time shows that the fractional change in the flux due to a change in $\phi$ is

$$
\frac{1}{\phi} \frac{d \phi}{d t}=(m-1) t^{-1} \text {. }
$$

For $m=0.66$ this corresponds to a fractional flux change of $-0.1 \% \mathrm{yr}^{-1}$, much smaller than the changes due to a change in cutoff energy.

For estimating the effect of the second term in Equation (7) we assume that $B^{2} \propto \rho V_{s}^{\beta}$, as suggested by Völk et al. (2005), with $\beta=2$ (Völk et al. 2005) or $\beta=3$ (Vink 2008; Bell 2004). Thus,

$$
B^{\Gamma_{0}} \propto\left(\rho V_{s}^{\beta}\right)^{\Gamma_{0} / 2} \propto\left(t^{-2 m} t^{(m-1) \beta}\right)^{\Gamma_{0} / 2}=t^{\Gamma_{0}[m(\beta-2)-\beta] / 2},
$$

where we have again assumed that the density structure declines as $1 / r^{2}$ and $r \propto t^{m}$.

The fractional change in $B^{\Gamma_{0}}$, and therefore the expected fractional flux change, is expected to be

$$
\frac{1}{B^{\Gamma_{0}}} \frac{d B^{\Gamma_{0}}}{d t}=\left(t^{-2 m} t^{(m-1) \beta}\right)^{\Gamma_{0} / 2}=t^{\Gamma_{0}(m(\beta-2)-\beta) / 2},
$$

corresponding to a flux change of $-0.45 \% \mathrm{yr}^{-1}$ for $\beta=2$, $m=0.66$, and $\Gamma_{0}=1.5$ or $-0.54 \% \mathrm{yr}^{-1}$ for $\beta=3$. These values are larger than those due to changes in the first term $(\phi)$, but smaller than the fractional changes predicted from changes in the cutoff energy alone. It is important to note that these values are only valid for the forward shock, for which we have a reasonable estimate of both the preshock density and $m$.

There are a number of caveats in the simple analysis above which may affect our result. For instance, Schure et al. (2010) point out that if non-steady-state acceleration effects are considered, the exponential cutoff may have a dependence that is different than that in Equation (2). Additionally, we are averaging over an ensemble of shock conditions over a span of 11 years; the shock conditions and power-law spectra will undoubtedly vary from position to position, and a sum of power-law spectra does not yield a power law (see also the discussion by Helder \& Vink 2008). In that respect, it is surprising that the hard X-ray spectrum of Cas A is best described by a power law (Renaud et al. 2006) with little evidence for a gradual steepening of the hard X-ray spectrum with energy as predicted by Equation (1).

It is also possible that not all the emission arises from synchrotron emitting electrons. Helder \& Vink (2008) point out that $\gtrsim 50 \%$ of the emission in the west is from synchrotron emission, and the remainder is likely from thermal continuum. 
The loss time for thermal electrons is much greater than the 2-4 year timescale seen in the $\mathrm{TeV}$ electrons. That is, over the relevant timescales, variations in thermal continuum emission will be much smaller than variations in the nonthermal emission and it is possible that the emission from the thermal component may increase over the approximately 11 year time span of our observations.

\section{SUMMARY AND CONCLUSION}

Our analysis of the 4.2-6 keV flux of Cas A shows a decline of $1.5 \% \mathrm{yr}^{-1}$ in the nonthermal X-ray emission across the entire SNR over 11 years, with a slightly larger decline rate of $1.9 \% \mathrm{yr}^{-1}$ from regions along the remnant's western limb. We find that, qualitatively, the observed spectral steepening and decline in flux can be explained by a simple model for changes in the electron cutoff energy which are brought about by a natural deceleration of the shock. We estimate an average deceleration of Cas A's forward shock velocity $\approx 20-70 \mathrm{~km} \mathrm{~s}^{-1} \mathrm{yr}^{-1}$.

The predicted decline in the nonthermal X-ray emission is about $4 \% \mathrm{yr}^{-1}$, which is nearly twice that observed. The difference between the predicted and observed decline might be explained by the fact that the $4.2-6.0 \mathrm{keV}$ continuum emission is not entirely due to synchrotron emission from shock accelerated electrons, but some of it is from the thermal continuum which does not evolve on the same timescale as the nonthermal emission. We have compared our results with models where the decline is a natural consequence of either a decrease in the number of particles entering the shock or a decrease in the efficiency of the shock to amplify the magnetic field and find that these models predict a decline of $\sim 0.1 \%-0.5 \% \mathrm{yr}^{-1}$, which is significantly less than the observed decline of $1.5 \%-1.9 \% \mathrm{yr}^{-1}$.

We thank Alexey Vikhlinin and Paul Plucinsky for useful discussions regarding the stability of the Chandra ACIS-S3 quantum efficiency. D.J.P. acknowledges support from the Chandra GO program through grant GO0-11094X as well as support from NASA contract NAS8-03060. J.V. is supported by a Vidi grant from the Netherlands Scientific Organization (NWO).

\section{REFERENCES}

Abdo, A. A., et al. 2010, ApJ, 710, L92

Aharonian, F., et al. 2001, A\&A, 370, 112

Aharonian, F., et al. 2007a, A\&A, 464, 235
Aharonian, F., et al. 2007b, ApJ, 661, 236

Albert, J., et al. 2007, A\&A, 474, 937

Allen, G. E., et al. 1997, ApJ, 487, L97

Anderson, M. C., \& Rudnick, L. 1996, ApJ, 456, 234

Baars, J. W. M., Genzel, R., Pauliny-Toth, I. I. K., \& Witzel, A. 1977, A\&A, 61,99

Bell, A. R. 2004, MNRAS, 353, 550

Bykov, A. M., Uvarov, Y. A., \& Ellison, D. C. 2008, ApJ, 689, L133

DeLaney, T., \& Rudnick, L. 2003, ApJ, 589, 818

DeLaney, T., Rudnick, L., Fesen, R. A., Jones, T. W., Petre, R., \& Morse, J. A 2004, ApJ, 613, 343

Ellison, D. C., \& Cassam-Chenaï, G. 2005, ApJ, 632, 920

Fesen, R. A., et al. 2006, ApJ, 645, 283

Ginzburg, V. L., \& Syrovatskii, S. I. 1965, ARA\&A, 3, 297

Gotthelf, E. V., Koralesky, B., Rudnick, L., Jones, T. W., Hwang, U., \& Petre, R. 2001, ApJ, 552, L39

Helder, E. A., \& Vink, J. 2008, ApJ, 686, 1094

Hughes, J. P., Rakowski, C. E., Burrows, D. N., \& Slane, P. O. 2000, ApJ, 528, L109

Humensky, T. B. 2008, in AIP Conf. Ser. 1085, High Energy Gamma-ray Astronomy, ed. F. A. Aharonian, W. Hofmann, \& F. Rieger (Melville, NY: AIP), 357

Hwang, U., \& Laming, J. M. 2003, ApJ, 597, 362

Katsuda, S., Petre, R., Mori, K., Reynolds, S. P., Long, K. S., Winkler, P. F., \& Tsunemi, H. 2010, ApJ, 723, 383

Keohane, J. W., Rudnick, L., \& Anderson, M. C. 1996, ApJ, 466, 309

Laming, J. M., \& Hwang, U. 2003, ApJ, 597, 347

Maeda, Y., et al. 2009, PASJ, 61, 1217

Malkov, M. A., \& O'C Drury, L. 2001, Rep. Prog. Phys., 64, 429

Patnaude, D. J., \& Fesen, R. A. 2007, AJ, 133, 147

Patnaude, D. J., \& Fesen, R. A. 2009, ApJ, 697, 535

Reed, J. E., Hester, J. J., Fabian, A. C., \& Winkler, P. F. 1995, ApJ, 440, 706

Reichart, D. E., \& Stephens, A. W. 2000, ApJ, 537, 904

Renaud, M., et al. 2006, ApJ, 647, L41

Reville, B., O’Sullivan, S., Duffy, P., \& Kirk, J. G. 2008, MNRAS, 386, 509

Reynolds, S. P. 1998, ApJ, 493, 375

Schure, K. M., Achterberg, A., Keppens, R., \& Vink, J. 2010, MNRAS, 406 2633

Shklovskii, I. S. 1960, SvA, 4, 355

The, L.-S., Leising, M. D., Kurfess, J. D., Johnson, W. N., Hartmann, D. H., Gehrels, N., Grove, J. E., \& Purcell, W. R. 1996, A\&AS, 120, 357

Tsujimoto, M., et al. 2011, A\&A, 525, A25

Uchiyama, Y., \& Aharonian, F. A. 2008, ApJ, 677, L105

Vink, J. 2008, in AIP Conf. Ser. 1085, High Energy Gamma-ray Astronomy, ed. F. A. Aharonian, W. Hofmann, \& F. Rieger (Melville, NY: AIP), 169

Vink, J., Kaastra, J. S., \& Bleeker, J. A. M. 1996, A\&A, 307, L41

Vink, J., \& Laming, J. M. 2003, ApJ, 584, 758

Vink, J., Maccarone, M. C., Kaastra, J. S., Mineo, T., Bleeker, J. A. M., PreiteMartinez, A., \& Bloemen, H. 1999, A\&A, 344, 289

Völk, H. J., Berezhko, E. G., \& Ksenofontov, L. T. 2005, A\&A, 433, 229

Willingale, R., Bleeker, J. A. M., van der Heyden, K. J., \& Kaastra, J. S. 2003, A\&A, 398, 1021

Willingale, R., Bleeker, J. A. M., van der Heyden, K. J., Kaastra, J. S., \& Vink, J. 2002, A\&A, 381, 1039

Zirakashvili, V. N., \& Aharonian, F. 2007, A\&A, 465, 695 